\title{
Analysis of TP53 gene expression and p53 level of human hypopharyngeal FaDu (HTB-43) head and neck cancer cell line after microRNA-181a inhibition
}

\author{
Y.K. Cheah ${ }^{1,2}$, R.W. Cheng ${ }^{1}$, S.K. Yeap ${ }^{2}$, C.H. Khoo ${ }^{1}$ and H.S. See ${ }^{3}$ \\ ${ }^{1}$ Faculty of Medicine and Health Sciences, University Putra Malaysia, \\ Serdang, Selangor, Malaysia \\ ${ }^{2}$ Institute of Bioscience, University Putra Malaysia, Serdang, \\ Selangor, Malaysia \\ ${ }^{3}$ Biomarketing Services (M) Sdn. Bhd., Bandar Menjalara, \\ Kuala Lumpur, Malaysia \\ Corresponding author: Y.K. Cheah \\ E-mail: ykcheah@medic.upm.edu.my
}

Genet. Mol. Res. 13 (1): 1679-1683 (2014)

Received September 7, 2013

Accepted November 8, 2013

Published January 22, 2014

DOI http://dx.doi.org/10.4238/2014.January.22.4

\begin{abstract}
The identification of new biomarkers for early detection of highly recurrent head and neck cancer is urgently needed. MicroRNAs (miRNAs) are small and non-coding RNAs that regulate cancer-related gene expression, such as tumor protein 53 (TP53) gene expression. This study was carried out to analyze TP53 gene expression using real-time PCR and to determine changes in intracellular p53 level by flow cytometry after downregulation of miRNA-181a miRNA inhibitor in the FaDu cell line. TP53 gene expression showed a 3 -fold increment and the p53 protein level was also increased in the miRNA-181a-treated cells. In conclusion,
\end{abstract}


miRNA-181a binds to the TP53 gene and inhibits its expression, decreasing the synthesis of $\mathrm{p} 53$.

Key words: Head and neck cancer; miRNA-181a; p53

\section{INTRODUCTION}

Almost all head and neck cancers are squamous cell carcinomas (HNSCC) that arise in the squamous epithelium (Rezende et al., 2010). HNSCC accounts for $6 \%$ of all cancer cases, representing the sixth most common type of cancer and cancer-related death worldwide (Argiris et al., 2008; Schaaij-Visser et al., 2010), with an average five-year survival rate of less than $50 \%$ for the last 30 years. Moreover, late stage diagnosis is the major reason that further reduces the survival rate of patients with regional or distant metastasis (Nurul-Syakima et al., 2011). Thus, the identification of new biomarkers to improve the current diagnosis of head and neck cancer is urgently needed for early detection and prognosis prediction to design better treatment (Twu et al., 2006). Like most other human cancers, the establishment of HNSCC also results from a multi-step process including activation of oncogenes and inactivation of tumor suppressor genes, which result from the accumulation of genetic and epigenetic alterations (Liu et al., 2009; Schaaij-Visser et al., 2010). In normal cells, p53 acts as the guardian of the genome by arresting the cell cycle and initiating apoptosis of cells with DNA damage through stimulation of the expression of downstream genes (Chien et al., 2003).

Nowadays, researchers in biomedical fields have expanded their interest in a noncoding, relatively small number of small genes with products $\sim 22$ nucleotide in length called microRNAs (miRNAs) (Liu et al., 2009). Many studies have profiled differential miRNA expression to distinguish cancer and normal cells for the identification of cancer markers (Nurul-Syakima et al., 2011). In addition, the finding of miRNAs that are differentially expressed in carcinoma samples suggests that miRNAs may be involved in cancer development. Therefore, to determine the function of every single miRNAs in head and neck cancers, we should study the genes, functions and pathway that are regulated by the miRNAs that are differentially expressed. One of the important pathways regulated by miRNAs is the p53 signaling pathway, which is responsible for tumor suppression activities such as DNA replication, repair and recombination to eliminate spontaneous and chemically induced DNA damage. The roles of p53 can be attributed to its ability to act as a sequence-specific transcription factor that coordinately activates and regulates the expression of multiple transcriptional targets to modulate various cellular processes (Morris, 2002; Nurul-Syakima et al., 2011). According to Nurul-Syakima et al. (2011), miRNA181a is upregulated in head and neck cancer, but the function of miRNA-181a in the p53 tumor suppressor pathway is still unknown. Moreover, it is hard to predict the target gene of miRNAs due to partial complementary binding of miRNAs and their target sequences (Tran et al., 2007; Seoudi et al., 2012), even with the aid of special prediction algorithms. Hence, miRNA functions, target genes and regulated pathways need to be validated by studies before being further exploited in cancer research (Nurul-Syakima et al., 2011). From this point of view, the target miRNA-181a may bind to mRNA of p53 and may also bind to other mRNAs. To understand this, the effect of miRNA-181a on p53 synthesis in 
downregulated $\mathrm{FaDu}(\mathrm{HTB}-43)$ hypopharyngeal cancer cells was evaluated using real-time PCR and flow cytometry in this study.

\section{MATERIAL AND METHODS}

The human hypopharyngeal FaDu (HTB-43) head and neck cancer cell line was used in this study. The cells were maintained in $89 \%$ medium (Iscove's Modified Dulbecco's medium), $10 \%$ fetal bovine serum (FBS), and 1\% antibiotic solution (100X penicillin and streptomycin) at $37^{\circ} \mathrm{C}$ in a humidified atmosphere with $5 \% \mathrm{CO}_{2}$. FaDu cells were divided into two groups: untreated cells (with complete medium only) and treated cells (with complete medium, transfection reagent and miRNA inhibitor). miRDIAN miRNA hairpin inhibitor (5 $\mu \mathrm{M})$, Dharmafect 1 Transfection reagent, serum-free medium and complete medium were added to prepare the transfection medium according to manufacturer instructions. After $48 \mathrm{~h}$, cells $(1 \mathrm{x}$ $10^{6}$ ) were harvested and total RNA was extracted using QIAzol Lysis Reagent (Qiagen, Hamburg, Germany) and the RNeasy Mini kit (Qiagen). cDNA was synthesized using a QuantiTect Reverse Transcription kit (Qiagen, Hamburg, Germany), according to manufacturer instruction. Real-time RT-PCR was performed using the Rotor-Gene SYBR Green PCR kit (Qiagen, Hamburg, Germany) in a Fast Real-time PCR System (Rotor Gene 6000). p53 mRNA was amplified using previously described primer pairs: 5'-AGA GTC TAT AGG CCC ACC CC -3'(forward) and 5'-GCT CGA CGC TAG GAT CTG AC-3' (reverse), and GAPDH 5'-CAT GGG GAA GGT GAA GGT CGG A-3' (forward) and 5'-TTG GCTCCC CCC TGC AAA TGA G -3' (reverse) (Miyachi et al., 2009). Relative target mRNA levels were determined using the delta-delta CT method (Schmittgen and Livak, 2008), with the formula given below (Livak and Schmittgen, 2001), and threshold value of 0.2, where the results were expressed in fold changes of treated cells compared with untreated cells.

$$
2^{-\Delta \Delta C T}=2^{-[(\text {miRNA } 533-\text { miRNA GADPH) - (normal p53 - normal GADPH) }]}
$$

Besides, transfected and non-transfected FaDu cells were harvested, fixed with $70 \%$ ethanol and stained with BD Via-Probe ${ }^{\mathrm{TM}}$ Cell Viability Solution (BD, USA) according to manufacturer instructions after $72 \mathrm{~h}$ of transfection. Cells were fixed using cold $70-80 \%$ ethanol. The expression of intracellular p53 was then analyzed using a BD Accuri 6 flow cytometer. Raji cells, showing constitutive expression of p53 (Zamai et al., 2002), were used as positive control in the flow cytometry study. Results for real-time PCR and flow cytometry are reported as means \pm SD from three independent experiments. One-way analysis of variance (ANOVA) followed by the Duncan test was used in this study and $\mathrm{P}<0.05$ was regarded as significant.

\section{RESULTS}

After $48 \mathrm{~h}$ of transfection, a 3-fold increase in TP53 gene expression was detected in transfected FaDu cells as compared to the non-transfected cells by real-time PCR (Table 1). On the other hand, flow cytometer analysis after $72 \mathrm{~h}$ demonstrated an increment in $\mathrm{p} 53^{+}$ cells from $0 \%$ in non-transfected FaDu cells to $83.4 \pm 0.55 \%$ in miRNA-181a knockdown FaDu cells. Positive control Raji cells also displayed a high percentage of p53+ $(88.8 \pm 1.1$ $\%$ ) cells (Figure 1). 
Table 1. Average Ct value and relative ratio of p53 expression on transfected and non-transfected FaDu cell.

\begin{tabular}{lccccccc}
\hline Name & Ct value 1 & Ct value 2 & Ct value 3 & Average Ct & $\Delta$ Ct & $\begin{array}{c}\Delta \Delta \text { Ct } \\
\text { Relative ratio } \\
\left(2^{\Delta \Delta C T}\right)\end{array}$ \\
\hline Transfected FaDu-p53 & 28.18 & 26.5 & 29.78 & 28.15 & 1.08 & -1.59 \\
Transfected FaDu-GAPDH & 26.55 & 26.59 & 28.09 & 27.08 & & 3.02 \\
Non-transfected FaDu-p53 & 28.64 & 28.67 & 29.69 & 29.00 & 2.67 & \\
Non-transfected FaDu-GAPDH & 26.49 & 26.06 & 26.44 & 26.33 & & \\
\hline
\end{tabular}

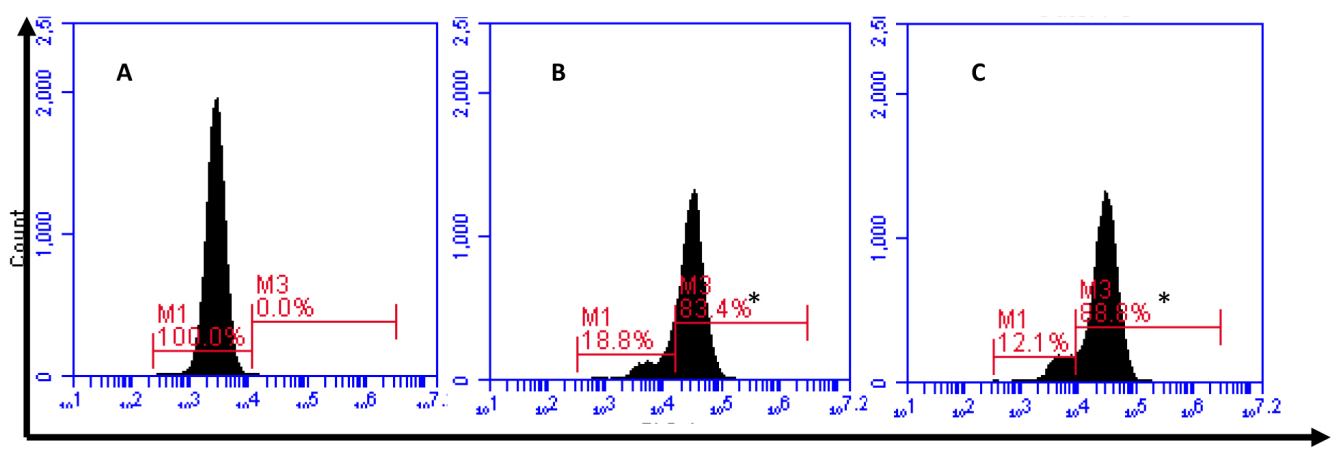

p53-PE

Figure 1. Intracellular deteciton of p53 on untreated control FaDu cells (A), treated $\mathrm{FaDu}$ cell $(\mathbf{B})$ and positive control Raji cell (C). M3 indicated p53 positively stained cell. ${ }^{*} \mathrm{P}<0.05$ compared to $\mathbf{A}$.

\section{DISCUSSION}

Recently, there have been many studies profiling differential miRNA expression in head and neck cancer. These results are very helpful in differentiating between tumors and normal tissues. In addition, some miRNAs have even been identified as biomarkers in cancer diagnosis and prognosis (Tran et al., 2007; Liu et al., 2009; Hui et al., 2010; Nurul-Syakima et al., 2011). The sequence of human miRNA-181a is 3'-UGAGUGGCUGUCGCAACUUAC AA-5', 23 nucleotides long (Wang et al., 2009). Upregulation of the oncogene miRNA-181a has been reported to be associated with downregulated p53 protein level in various cancer cells such as squamous head and neck carcinoma, HPV $(+)$, multiple myeloma and MCF-7 cell line (Shi et al., 2008; Seoudi et al., 2012).

Recent findings indicate that mRNA degradation can occur even though the binding between miRNAs and their target genes share only partial sequence complementarity. However, it is unclear whether translational processes are inhibited preceding destabilization of target genes or vice versa (Esquela-Kerscher and Slack, 2006). From the results of real time PCR, gene expression of the TP53 gene was increased around 3-fold after miRNA-181a inhibition. This indicated that miRNA-181a binding may cause mRNA of TP53 gene to be destabilized or degraded at the post-transcriptional level. Flow cytometry analysis demonstrated that the p53 protein level of transfected FaDu cells increased significantly compared with untreated FaDu cells. These results indirectly proved that miRNA-181a bound to mRNA of the TP53 gene and inhibited its translation, while the presence of miRNA-181a inhibitor successfully restored p53 synthesis.

Since FaDu cells possess only mutant p53, functional studies such as cell cycle and 
apoptosis analysis should be carried out in the future to ensure the functionality of restored p53 after knockdown of miRNA-181a. Furthermore, further studies can also be performed to examine if there are any other factors that may affect or act together with miRNA-181a to inhibit the post-transcriptional activities of $\mathrm{p} 53$, since a wide range of miRNAs besides miR181a could control TP53 at the post-transcriptional level and because cancer is usually caused by multiple factors (Seoudi et al., 2012).

In conclusion, miRNA-181a bound to mRNA of the TP53 gene and affected the posttranscriptional processing of the TP53 gene. p53 mRNA and protein levels were increased after suppression of miRNA-181a. Therefore, miRNA-181a is considered an oncogene in head and neck cancer. This will be a novel biomarker that may be a useful in the early diagnosis, treatment, and prognosis of head and neck cancer.

\section{ACKNOWLEDGMENTS}

Research supported by the Universiti Putra Malaysia Research University Grant Scheme (\#04-05-10-1109RU) and the National Cancer Council Malaysia. The authors thank the Cell Signaling Laboratory and Physiology Laboratory, Universiti Putra, Malaysia, for providing technical assistance and guidance in mammalian cell culture and cell imaging technique.

\section{REFERENCES}

Argiris A, Karamouzis MV, Raben D and Ferris RL (2008). Head and neck cancer. Lancet 371: 1695-1709.

Chien CY, Huang CC, Cheng JT, Chen CM, et al. (2003). The clinicopathological significance of p53 and p21 expression in squamous cell carcinoma of hypopharyngeal cancer. Cancer Lett. 201: 217-223.

Esquela-Kerscher A and Slack FJ (2006). Oncomirs - microRNAs with a role in cancer. Nat. Rev Cancer 6: 259-269.

Hui AB, Lenarduzzi M, Krushel T, Waldron L, et al. (2010). Comprehensive MicroRNA profiling for head and neck squamous cell carcinomas. Clin. Cancer Res. 16: 1129-1139.

Liu X, Chen Z, Yu J, Xia J, et al. (2009). MicroRNA profiling and head and neck cancer. Comp. Funct. Genomics 837514.

Livak KJ and Schmittgen TD (2001). Analysis of relative gene expression data using real-time quantitative PCR and the $2^{-\Delta \Delta C T}$ Method. Methods 25: 402-408.

Miyachi M, Kakazu N, Yagyu S, Katsumi Y, et al. (2009). Restoration of p53 pathway by nutlin-3 induces cell cycle arrest and apoptosis in human rhabdomyosarcoma cells. Clin. Cancer Res. 15: 4077-4084.

Morris SM (2002). A role for p53 in the frequency and mechanism of mutation. Mutat. Res. 511: 45-62.

Nurul-Syakima AM, Yoke-Kqueen C, Sabariah AR, Shiran MS, et al. (2011). Differential microRNA expression and identification of putative miRNA targets and pathways in head and neck cancers. Int. J. Mol. Med. 28: 327-336.

Rezende TM, de Souza FM and Franco OL (2010). Head and neck cancer: proteomic advances and biomarker achievements. Cancer 116: 4914-4925.

Schaaij-Visser TB, Brakenhoff RH, Leemans CR, Heck AJ, et al. (2010). Protein biomarker discovery for head and neck cancer. J. Proteomics 73: 1790-1803.

Schmittgen TD and Livak KJ (2008). Analyzing real-time PCR data by the comparative C(T) method. Nat. Protoc. 3: 11011108.

Seoudi AM, Lashine YA and Abdelaziz AI (2012). MicroRNA-181a - a tale of discrepancies. Expert. Rev. Mol. Med. 14: e5.

Shi L, Cheng Z, Zhang J, Li R, et al. (2008). hsa-mir-181a and hsa-mir-181b function as tumor suppressors in human glioma cells. Brain Res. 1236: 185-193.

Tran N, McLean T, Zhang X, Zhao CJ, et al. (2007). MicroRNA expression profiles in head and neck cancer cell lines. Biochem. Biophys. Res. Commun. 358: 12-17.

Twu CW, Jiang RS, Shu CH and Lin JC (2006). Association of p53 codon 72 polymorphism with risk of hypopharyngeal squamous cell carcinoma in Taiwan. J. Formos. Med. Assoc. 105: 99-104.

Wang X, Gocek E, Liu CG and Studzinski GP (2009). MicroRNAs181 regulate the expression of p27Kip1 in human myeloid leukemia cells induced to differentiate by 1, 25-dihydroxyvitamin D3. Cell Cycle 8: 736-741.

Zamai L, Canonico B, Gritzapis A, Luchetti F, et al. (2002). Intracellular detection of Bcl-2 and p53 proteins by flow cytometry: comparison of monoclonal antibodies and sample preparation protocols. J. Biol. Regul. Homeost. Agents 16: 289-302. 